\title{
Extended total arch replacement by means of the open stent-grafting method to treat intimal tears after transluminal stent-graft placement for a ruptured acute type B aortic dissection
}

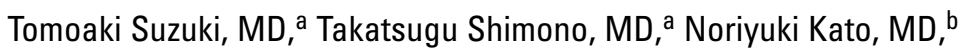
Uhito Yuasa, MD, ${ }^{a}$ Koji Onoda, MD, ${ }^{a}$ Tadanori Hirano, MD, ${ }^{b}$ Kan Takada, MD, ${ }^{b}$ and Isao Yada, MD, ${ }^{\text {a }}$ Tsu, Japan

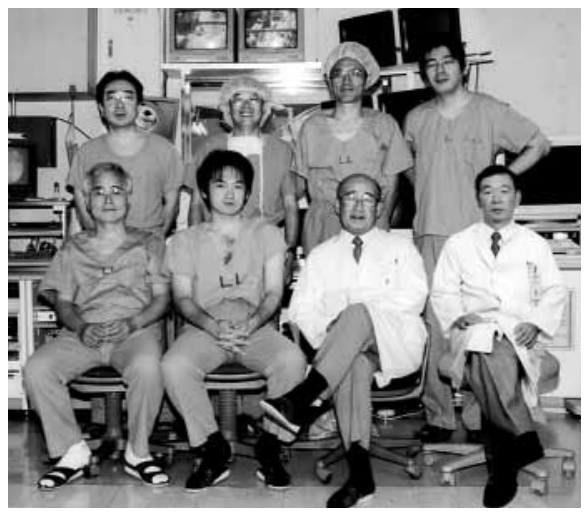

Front row (left to right): Drs Hirano, Suzuki, Yada, Takeda. Back row (left to right): Drs Onoda, Shimono, Kato, Yuasa.
$\mathrm{E}$ ndovascular stent grafting is an effective modality for the treatment of an acute aortic dissection and is less invasive than implantation of a vascular prosthesis. ${ }^{1-3}$ We have performed endovascular stent grafting in 22 cases of acute aortic dissection with satisfactory initial results; however, a new, secondary intimal tear after stent-graft placement is a severe complication and may require surgical repair. We report the successful treatment of a secondary intimal tear after endovas-

From the Departments of Thoracic and Cardiovascular Surgery a and Radiology, ${ }^{\mathrm{b}}$ Mie University School of Medicine, Tsu, Japan.

Received for publication May 11, 2001; accepted for publication May 25, 2001.

Address for reprints: Takatsugu Shimono, MD, Department of Thoracic and Cardiovascular Surgery and Department of Radiology, Mie University School of Medicine, 2-174 Edobashi, Tsu, Mie, 514-8507, Japan (E-mail: simonot@clin.medic.mie-u.ac.jp).

J Thorac Cardiovasc Surg 2002;123:354-6

Copyright $\odot 2002$ by The American Association for Thoracic Surgery

$0022-5223 / 2002 \$ 35.00+0 \quad \mathbf{1 2 / 5 4} / \mathbf{1 1 7 5 3 3}$

doi: $10.1067 / \mathrm{mtc} .2002 .117533$ cular stent grafting for an acute type B aortic dissection with an open procedure using the open stent method. ${ }^{4}$

\section{Clinical Summary}

A 75-year-old man was referred to our hospital on an emergency basis from a satellite hospital with the diagnosis of contained rupture of an acute type B aortic dissection. On arrival, he was conscious and in hemodynamically stable condition. Emergency digital subtraction angiography (DSA) showed an entry $2 \mathrm{~cm}$ distal to the origin of the left subclavian artery (Figure 1). The length of the proximal landing zone was $1.5 \mathrm{~cm}$, and its diameter was $3.5 \mathrm{~cm}$; the length of the entry tear was $1 \mathrm{~cm}$. We performed endovascular stent grafting according to our inclusion criteria for an acute type B aortic dissection. ${ }^{5}$ Transfemoral endovascular grafting under general anesthesia was performed by means of a previously reported technique. ${ }^{3,4}$ First, the stent graft was placed from 1 to $8.5 \mathrm{~cm}$ distal to the orifice of the left subclavian artery. However, a type I intimal leak proximal to the graft was noted, and therefore a second stent graft was added from the orifice of the left subclavian artery extending into the first stent graft in stent-graft-in-stentgraft fashion. Postprocedure DSA showed no leak. The first graft was covered with polyester, and the second was an expanded polytetrafluoroethylene graft. Contrast-enhanced computed tomography performed 1 month after stent grafting showed a reduction in 


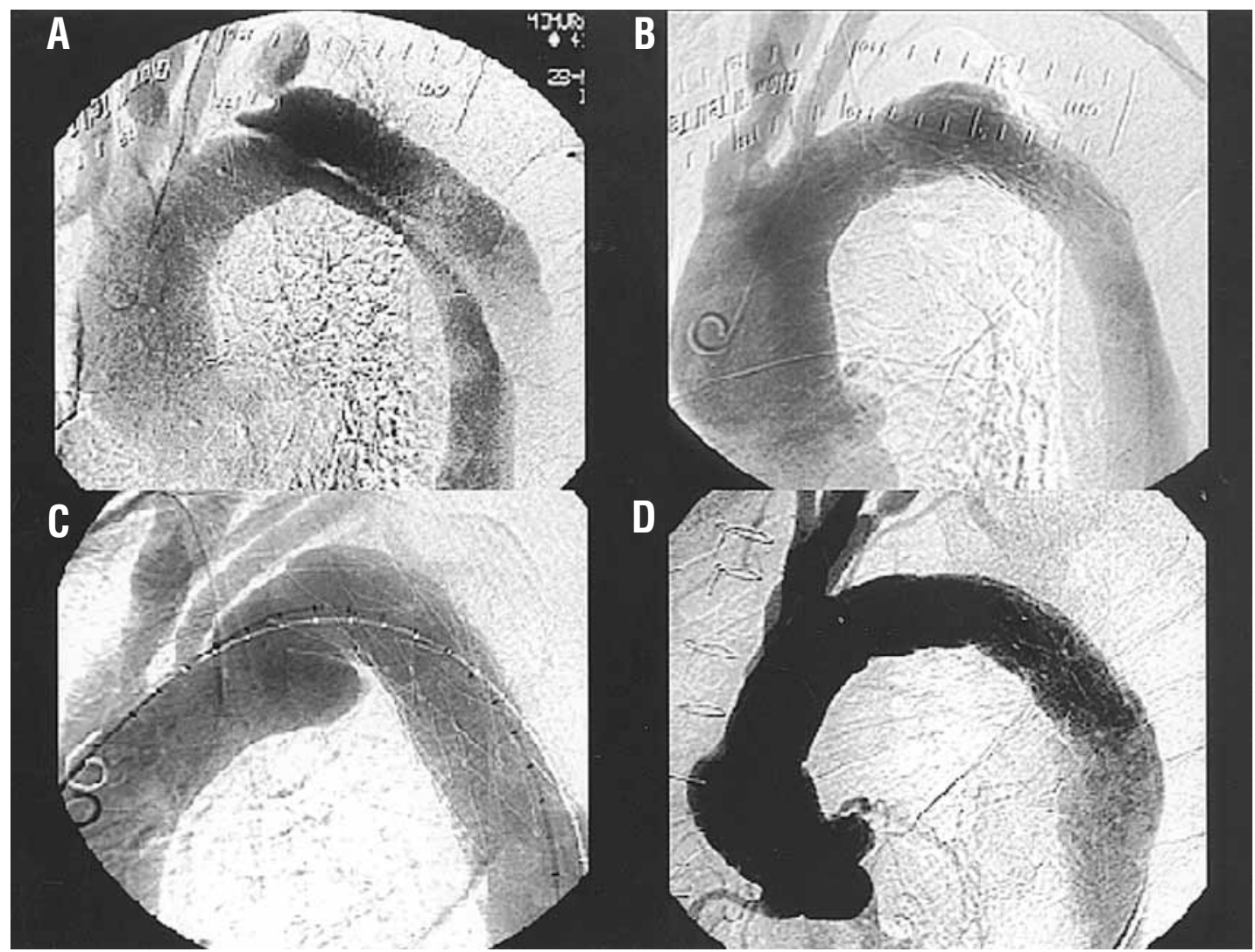

Figure 1. A, DSA showing the intimal tear at the proximal descending thoracic aorta $2 \mathrm{~cm}$ distal to the origin of the left subclavian artery and extravasation of blood. B, DSA 1 month after the stent-graft repair confirming no leakage from the stent graft. C, DSA 3 months after the stent-graft repair showing the intimal tear in the area of the proximal portion of the stent graft with aneurysm formation. D, DSA 2 weeks after the extended total arch replacement with the open stent method confirming no distal leakage from the stent graft.

the size of the false lumen, with no leak. DSA confirmed the absence of leakage and migration of the stent graft. The postoperative course was uneventful.

Three months after endovascular grafting, computed tomography revealed a new intimal tear in the area of the proximal portion of the stent graft with aneurysm formation. Because the proximal landing zone was inadequate to perform additional endovascular grafting, extended total arch replacement with the open stent-graft method was performed 4 months after endovascular grafting.

After conventional median sternotomy, the arch vessels were dissected, and the aortic arch was exposed. The proximal descending aorta was $5 \mathrm{~cm}$ in diameter. Cardiopulmonary bypass was established through cannulation of the right femoral artery and the right atrium. The aorta between the left common carotid artery and the left subclavian artery was opened under circulatory arrest by means of selected brain perfusion at $20^{\circ} \mathrm{C}$. The intima at the proximal end of the stent graft was dissected hemicircumferentially. The proximal stent graft, which had been added after placement of the first stent graft, was removed easily, and a new stent graft was inserted into the first stent graft. This was a 7-cm long Dacron graft with 2 stainless-steel $\mathrm{Z}$ stents. The proximal end of the new stent graft was sutured to the aortic wall, and the branched graft was anastomosed to the proximal aspect of the stent graft and aortic wall. The branched graft was anastomosed to the 3 arch vessels and ascending aorta in an end-to-end fashion, and the extended total arch replacement was completed (Figure 2). The postoperative course was uneventful, and an aortogram performed 2 weeks after the operation revealed satisfactory replacement of the ascending through descending aorta, with no leakage or stenosis. The patient was discharged 3 weeks after the operation.

\section{Comment}

Endovascular stent grafting for acute aortic dissection is not a common treatment. Our group and the Stanford group have used this procedure to acute aortic dissection, and we believe that it may be an attractive alternative to open operations in patients with acute aortic dissection. ${ }^{1}$ Since 1997 , we have performed endovascular stent grafting in 22 cases of acute aortic dissection, including 2 ruptured aneurysms, with satisfactory initial results. However, a secondary intimal tear caused by stent-graft placement is a concern, particularly in cases of acute aortic dissection because the intima is weak. We have encountered 2 cases of acute dissection in which new intimal tears developed at the distal or proximal end of the stent graft. Two methods of treatment are 

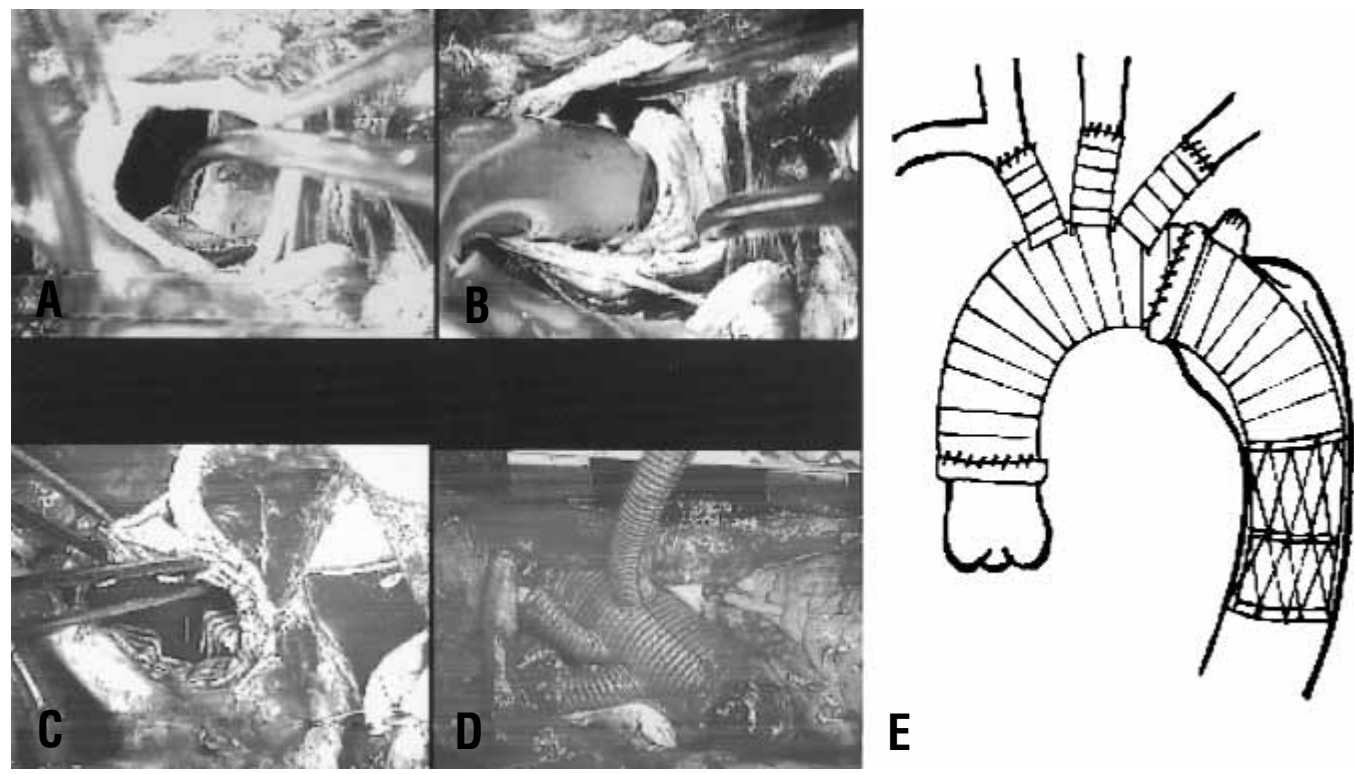

Figure 2. A, The aorta is transected between the left common carotid artery and the left subclavian artery. The previous inserted stent graft can be seen. B, A new stent graft is inserted into the previous stent graft. C, The proximal end of the new stent graft was sutured to the aortic wall. D, The branched graft was anastomosed to the 3 arch vessels and ascending aorta in an end-to-end fashion, and the extended total arch replacement was completed. E, Schema of the extended total arch replacement with the open stent-graft method in stent-graft-in-stent-graft fashion.

applicable for this complication. The first is additional endovascular grafting, and the other is to perform a conventional open procedure. In our case the secondary intimal tear developed at the proximal end of the stent graft 3 months after the procedure, and the proximal landing zone was insufficient to perform additional stent grafting; therefore, an open procedure was the only alternative. The proximal stent graft was removed easily without migration of the distal graft. Extended total arch replacement with the open stent method was performed easily, and the dissecting aneurysm was excluded completely. Our procedure is useful for the treatment of new intimal tears and useful to manage proximal leaks from the distal arch to the proximal descending aorta. We believe that secondary intimal tears are the result of poor flexibility of the stent graft, and therefore more flexible stent grafts must be developed to prevent this complication.

\section{References}

1. Dake MD, Kato N, Mitchell RS, Semba CP, Razavi MK, Shimono T, et al. Endovascular stent-graft placement for the treatment of acute aortic dissection. $N$ Engl J Med. 1999;340:1546-52.

2. Dake MD, Miller DC, Mitchel RS, Semba CP, Moore KA, Sakai T. The "first generation" of endovascular stent-grafts for patients with aneurvsms of the descending thoracic aorta. $J$ Thorac Cardiovasc Surg. 1998;116:689-704.

3. Shimomo T, Kato N, Tokui T, Onoda K, Hirano T, Takeda K, et al Endovascular stent-graft repair for acute type A aortic dissection with an intimal tear in the descending aorta. J Thorac Cardiovasc Surg. 1998;116:171-3.

4. Kato M, Matsuda T, Kaneko M, Ueda T, Kuratani T, Yoshioka Y, et al. New graft-implanting aneurysm or dissection with a stented graft. Circulation. 1996;94(Suppl):II-188-93.

5. Shimono T, Fuyuhiko Y, Adachi K, Kato N, Onoda K, Sinpo H, et al. Feasibility of endovascular stent grafting for thoracic aortic aneurysm, especially in acute aortic dissection. Jpn J Vasc Surg. 1999;4:473-80. 